\title{
Mortalidad atribuible al consumo de tabaco en México
}

\author{
Pablo Kuri-Morales MD. MSc, ${ }^{(1)}$ Jesús A legre-Díaz MD. EC G.,1) \\ Ma. del Pilar Mata-Miranda MD. EEA., (1) Mauricio Hernández-Á vila MD. MSc. D Ph. ${ }^{(2)}$
}

\begin{abstract}
Kuri-Morales P,Alegre-Díaz J, Mata-Miranda MP, Hernández-Avila M. Mortalidad atribuible al consumo de tabaco en México.

Salud Publica Mex 2002;44 supl 1:S29-S33. El texto completo en inglés de este artículo está disponible en: http://www.insp.mx/salud/index.html
\end{abstract}

\section{Resumen}

Objetivo. Estudiar la asociación entre defunciones registradas en la delegación Coyoacán y consumo de tabaco y alcohol. Material y métodos. Estudio de mortalidad proporcional y determinación de fracción atribuible en una cohorte de sujetos de la ciudad de México, D.F., México. Se reunió información de todas las defunciones de 1998 captadas a través del Sistema Estadístico y Epidemiológico de las D efunciones (SEED) en la delegación C oyoacán, clasificándolas por diagnóstico en enfermedades del sistema circulatorio (ESC), sistema respiratorio (EPO C), neoplasias (N EO). Se encuestó a familiares de los fallecidos sobre consumo de tabaco y alcohol. Resultados. Fumar los últimos 10 años de vida fue significativo para ESC en hombres de 70 años y más (RM : 2.06, IC 95\%=1.18-3.58); y continuar fumando el último año de vida fue significativo paraN EO y para EPOC en mujeres de 70 años y más (N EO = RM: 7.24, IC 95\%=1.71-30.53; ESR= RM : 4.82, IC 95\%=1.41-16.50). En el modelo de regresión para ESC, las personas con tabaquismo intenso tuvieron una posibilidad 0.83 veces mayor de fallecer por ESC, y el RA de esta variable para las ESC en la población general fue $45 \%$. $(\mathrm{RM}=1.83 ;$ IC $95 \%=1.1-2.8 \mathrm{p}<0.01)$. Conclusiones. La exposición a tabaco es responsible de la elevada incidencia y mortalidad de enfermedades del sistema circulatorio y respiratorio, así como de neoplasias, en una muestra poblacional de sujetos de la ciudad de México. El texto completo en inglés de este artículo está disponible en:http://www.insp.mx/ salud/index.html

Palabras clave: enfermedades crónicas; tabaquismo; riesgo atribuible; México

\author{
Kuri-Morales P,Alegre-Díaz J, \\ Mata-Miranda MP, Hernández-Avila M. \\ Mortality attributable to tobacco \\ smoking in Mexico. \\ Salud Publica Mex 2002;44 suppl 1:S29-S33. \\ The English version of this paper \\ is available at: http://www.insp. mx/salud/index.html
}

\begin{abstract}
A bstract
Objective.To study the mortality attributable to tobacco and alcohol consumption. Material and Methods. Deaths occurring in 1998, abstracted from the mortality statistics registry in the Coyoacan District of Mexico City, were classified by cause as: C ardiovascular D iseases, Respiratory Diseases, and $\mathrm{N}$ eoplasias. Relatives of the deceased were inter viewed to answer a questionnaire on tobacco smoking and alcohol drinking. Results Smoking during the last ten years of life was significantly associated with cardiovascular disease in men aged 70 years or older $(0 \mathrm{R} 2.06,95 \% \mathrm{CI}$ 1.18-3.58). Persistent smoking in the last year of life was significantly associated with deaths from neoplasias and respiratory diseases in women aged 70 years or older (OR: 7.24, 95\% CI 1.71-30.53; O R: 4.82 95\% CI 1.41-16.50, respectively). The regression model for cardiovascular disease showed that subjects with intense tobacco consumption were almost twice as likely to die from cardiovascular disease $(0 \mathrm{R} 1.83,95 \% \mathrm{Cl} 1.1-2.8, \mathrm{p}<0.01)$. The population attributable risk for cardiovascular disease was $45 \%$. Conclusions. Tobacco smoking is an important predictor of dead among the elderly in Mexico. Tobacco control programs should also be targeted to the elderly. The English version of this paper is available at: http:// www.insp.mx/salud/index.html
\end{abstract}

Key words: chronic diseases; to bacco consumption; attributable risk; Mexico

(1) Dirección General de Epidemiología, Secretaría de Salud, México, D.F., México.

(2) Centro de Investigación en Salud Poblacional, Insituto N acional de Salud Pública, Cuernavaca, Morelos, México.

Fecha de recibido: 1 de febrero de 2002 - Fecha de aceptado: 24 de abril de 2002

Solicitud de sobretiros: Dr. Jesús Algre Díaz. Dirección General de Epidemiología, Secretaría de Salud. Francisco de P. Miranda 177, $5^{\circ}$ piso, colonia Lomas de Plateros, Delegación Alvaro 0 bregón, 01480, México, D.F., México

Correo electrónico: jalegre@ epi.org.mx 
a Organización Mundial de la Salud (OMS) señala que el tabaco es la causa de mortalidad evitable más importante en el mundo. Que el consumo de tabaco es responsable de más de 3 millones de muertes al año y que tiene además, repercusiones sociales y económicas muy importantes. ${ }^{1}$ De acuerdo con datos de la OMS, se estima que actualmente hay cerca de 1100 millones de fumadores alrededor de todo el mundo; de ellos 300 millones viven en países desarrollados, y el resto, son ciudadanos de países en desarrollo. ${ }^{2,3}$ En México los fumadores aumentaron de 9.2 millones en 1988 a 14.3 millones en 1998 y se estima que anualmente ocurren más de 6 mil muertes por cáncer de pulmón, y más de 12 mil por cardiopatía, directamente causadas por el hábito de fumar. ${ }^{4}$

La evidencia científica que indica que el tabaquismo provoca aumento en la frecuencia de enfermedad pulmonar obstructiva crónica, enfisema, cáncer de pulmón y enfermedad cardiovascular es indiscutible. ${ }^{5}$ Igualmente, se han reportado en la literatura médica y económica un buen número de estudios en los que se documenta la importante carga económica que imponen a los sistemas de salud las enfermedades asociadas con el consumo de tabaco. ${ }^{6}$ Sin embargo, la extrapolación y aplicación directa de los estudios reportados a países como México es limitada, ya que la mayor parte de los trabajos se han realizado en países desarrollados, cuyas poblaciones difieren de manera importante de la nuestra, en sus hábitos de fumar, en estructura de edad, así como en la presencia de otros factores biológicos y sociales importantes. Además, no son comparables ${ }^{6}$ tanto los costos como los esquemas de tratamiento y la accesibilidad a los sistemas de salud encargados de proveer la atención médica requerida en cada país.

En México, se desconocen tanto la fracción atribuible de enfermedad asociada con el tabaco, como los costos que éstas ocasionan al sistema de salud. Como un primer paso para estimar el costo de la epidemia de tabaquismo en el país, en este trabajo hemos estimado las fracciones de mortalidad atribuibles a tabaco, utilizando un diseño de mortalidad proporcional que se basó en las muertes que ocurrieron en la delegación Coyoacán de la Ciudad de México durante 1998.

\section{Material y métodos}

Se trata de un estudio de mortalidad proporcional y determinación de fracción atribuible en una cohorte de sujetos de la ciudad de México, D.F., México. La población en estudio estuvo constituida por los residentes de la delegación Coyoacán que fallecieron durante el mismo año. La muestra estudiada se conformó con todas las muertes ocurridas en residentes de esta delegación du- rante 1998. Dado que las muertes que ocurren en sujetos menores de 35 años raramente están relacionadas con el consumo de cigarrillos, la población elegible para ser incluida en el estudio se limitó a mayores de 35 años. Como fuente de información primaria se utilizó el Sistema Estadístico y Epidemiológico de las Defunciones (SEED) de la misma delegación, además la información se complementó identificando las muertes de residentes de la zona en estudio que fueron reportadas en otras delegaciones. Esto último se llevo a cabo mediante una revisión de todos los certificados reportados en el Distrito Federal en 1998. Los certificados fueron revisados por un médico y un codificador. Se verificó la correcta anotación de los diferentes diagnósticos de defunción incluyendo tanto la causa básica como los estados morbosos asociados. En cada diagnóstico se cotejó su respectiva codificación según las reglas de la Clasificación Internacional de Enfermedades (CIE-10). ${ }^{7}$ En el 80.3\% ( $\left.\mathrm{n}=2557\right)$ de los certificados revisados la información registrada en el SEED y la reportada en el certificado de defunción coincidieron. El 19.7\% de los certificados restantes fueron excluidos del estudio debido a que no se encontraron, o por haber diferencias entre lo registrado en el certificado y la base de datos del SEED en cuanto a la edad del sujeto, la residencia habitual o la identificación.

Una vez seleccionados los sujetos de estudio se acudió al último domicilio registrado en el certificado de defunción y se invitó a participar a un familiar cercano que al menos hubiera convivido con el sujeto de estudio durante el último año de vida. A los sujetos que aceptaron participar se les pidió responder un cuestionario, el cual indagaba sobre las características de consumo de tabaco y tabaquismo pasivo, sobre las características de consumo de alcohol, así como consumo de tabaco en relación con el consumo de bebidas alcohólicas y sobre otros factores sociodemográficos básicos. La entrevista se hizo al cónyuge en $15.8 \%$ de los casos, a los hijos en $34.3 \%$; a los nietos en $11.7 \%$; y a otro familiar en $13 \%$ de las ocasiones.

La encuesta se llevó a cabo con sistemas basados en lectores ópticos de código de barras, y éstos fueron impresos a través del programa Universal Data Language versión 3.5.

\section{Padecimientos estudiados}

Los padecimientos estudiados fueron enfermedades del sistema circulatorio (I00-I99), enfermedades del sistema respiratorio (J00-J99) y neoplasias (C00-D48), el resto de los diagnósticos constituyeron el grupo de comparación o referencia (cuadro I).

El tabaquismo se definió como un consumo de tabaco consuetudinario de por lo menos un año durante la 
vida del sujeto de estudio. Se preguntó al entrevistado si el sujeto de estudio fumaba o fumó durante los 10 años previos a su fallecimiento, si lo hacía el año antes de su fallecimiento, en qué presentación consumía el tabaco, si consumía cigarros con o sin filtro, la frecuencia con la que fumaba, el número de cigarros, en cada ocasión. Además se indagó sobre tabaquismo pasivo en el mismo hogar; esto último se investigó preguntando si había alguna otra persona que fumara en el interior del hogar de una manera consuetudinaria.

\section{Resultados}

La base de datos obtenida en campo se depuró y analizó mediante una serie de códigos diseñados en plataforma Fox-pro 2.6 para Windows 95 y con el paquete estadístico SPSS10; los análisis que se utilizaron para el modelo de regresión y con el que se estimaron las fracciones atribuibles, se basaron en lo publicado por Whittemore. ${ }^{8}$

La asociación entre tabaco y riesgo de morir por las diferentes enfermedades estudiadas se evaluó ajustando por edad y género; la edad se transformó en una variable dicotómica, dividiéndola en dos grupos de 35 a 69 años de edad, y de 70 años o mayores.

\section{Cuadro I \\ Diagnósticos Utilizados para definiR A Los Casos Según la CIE-10. Ciudad de México, 1998}

\begin{tabular}{|c|c|c|}
\hline \multirow[t]{3}{*}{$\begin{array}{l}\text { Enfermedades del } \\
\text { sistema circulatorio }\end{array}$} & Neoplasias & $\begin{array}{l}\text { Enfermedades del } \\
\text { sistema respiratorio }\end{array}$ \\
\hline & $(\mathrm{C} 00.0-\mathrm{C} 06.9)$ & $(J 43.1-J 44.9)$ \\
\hline & Tumores de los labios & Enfisema \\
\hline$(\mid 20.0-125.9)$ & (C 09.0-C 14.0) & \\
\hline \multirow[t]{2}{*}{ Angina de pecho } & Tumor maligno & \\
\hline & de la amígdala & \\
\hline$(160.0-166.9)$ & (C 15.0-C 15.5) & \\
\hline Hemorragias & Tumor maligno & \\
\hline cerebrales & de esófago & \\
\hline$(170.0-171.9)$ & (C25.0-C 25.9) & \\
\hline Aterosclerosis & Tumor maligno & \\
\hline & de páncreas & \\
\hline$(174.0-174.9)$ & (C 34.0-C 34.9) & \\
\hline \multirow{2}{*}{$\begin{array}{c}\text { Embolia y trombosis } \\
\text { arteriales }\end{array}$} & Tumor maligno & \\
\hline & de bronquios y pulmón & \\
\hline (C 39.0-C 39.9) & (C 64.0-C 67.9) & \\
\hline Tumor maligno & Tumor maligno & \\
\hline de sistema respiratorio & de riñón & \\
\hline
\end{tabular}

Fuente: Clasificación Internacional de Enfermedades CIE-10
Basados en la información provista por el SEED, de 3138 defunciones esperadas en la delegación Coyoacán para el año 1998, se identificaron 2557 defunciones correspondientes al grupo de 35 años y más; de éstas sólo en 1158 (45.3\%) se obtuvo información completa. El promedio de edad de la población estudiada fue de 71 años $(D E \pm 15.34), 47 \%$ ( $n=1158)$ correspondieron al género masculino. El $67 \%$ de la población estudiada contaba con estudios de primaria completa o menos.

El 30\% de los fallecidos fumó durante los últimos 10 años de su vida y $18 \%$ de la población fumó también durante el año antes de fallecer. El $75 \%$ de los fumadores consumía cigarrillos con filtro, $81.6 \%$ fumaba a diario y el resto ocasional, semanal o mensualmente. La mediana de consumo de cigarrillos reportada fue de cinco al día, con un rango intercuartilar de 18. En 38\% de los participantes se identificó también tabaquismo pasivo.

En relación con el consumo de bebidas alcohólicas, se encontró que $47.5 \%$ nunca consumió alcohol durante el año antes de fallecer, mientras que $10.4 \%$ lo consumieron tres veces por semana o con mayor frecuencia.

En el modelo de regresión logística para enfermedades del SC (cuadro II), se encontró que las personas que tuvieron positiva la variable tabaquismo intenso tuvieron una posibilidad 0.83 veces mayor de fallecer por enfermedades del SC, y el riesgo atribuible de esta variable para las enfermedades del SC en la población general fue de $45 \%$. $(R M=1.83 ; p<0.01$; IC $95 \%=1.1-2.8)$.

Cuando se estratificó por edad y sexo, este mismo modelo en los hombres mostró que las personas con la variable tabaquismo intenso positiva tuvieron una posibilidad 1.5 veces mayor de fallecer por enfermedades del SC, y el riesgo atribuible de esta variable para las enfermedades del SR en la población general fue de $60 \%$. $(R M=2.5 ; p=0.001 ;$ IC $95 \%=1.4-4.4)$.

En hombres de 70 años o más, se encontró que los decesos debidos a cardiopatía isquémica tenían una estimación de riesgo de morir 2 veces mayor en aquellos sujetos quienes fumaron 10 años antes de morir, que en los que no lo hicieron $(R M=2.06 ; p=0.01$, IC $95 \%=1.18$, 3.58). Y aquellos que continuaron fumando un año antes de morir tuvieron un riesgo de hacerlo por cardiopatía 1.9 veces mayor que el de quienes que no lo hicieron $(R M=1.9 ; p=0.03$, IC $95 \%=1.05,3.70)$.

En el mismo modelo aplicado a las mujeres se encontró que ninguna variable se asoció significativamente con el riesgo de morir por enfermedades del SC.

En el modelo de regresión para neoplasias, se encontró que las personas que tuvieron positiva la variable tabaquismo intenso tuvieron una posibilidad 1.88 veces mayor de fallecer por neoplasias, y el riesgo atribuible de esta variable para las neoplasias en la 
población general fue de $64 \%$. $(R M=2.88 ; p=0.01$; IC $95 \%=1.1-7.02$ ).

Cuando se estratificó por edad y sexo, este mismo modelo en los hombres mostró que ninguna variable fue significativa como factor de riesgo para fallecer por alguna neoplasia. En las mujeres, se encontró que las personas que tuvieron positiva la variable tabaquismo intenso tuvieron una posibilidad 6.1 veces mayor de fallecer por neoplasias, y el riesgo atribuible de esta variable para las neoplasias en la población general fue de $85 \%$. $(R M=7.1 ; p=0.003$; IC $95 \%=1.9-25.8)$.

Al estratificar por edad y sexo, en las mujeres de 35 a 69 años de edad se encontró un riesgo aumentado para fallecer por neoplasias, en aquellos casos donde aún fumaban un año antes de fallecer hasta 14 veces mayor que en aquellas que no fumaron el último año $(R M=14.17 ; p<0.01$, IC $95 \%=1.23,162.59)$. Este patrón se repitió en mujeres de 70 años y más, quienes tuvieron un riesgo aumentado de morir por neoplasias siete veces mayor en aquellas que fumaron hasta el año previo antes de fallecer, que en quienes no fumaron el último año de vida $(R M=7.24 ; p=0.002$, IC $95 \%=1.71,30.53)$.

\section{Cuadro II \\ Riesgo de MORTALIDAD DE TABAQUiSMO Y enfermedades Crónicas. Ciudad de México, 1998}

\begin{tabular}{|c|c|c|c|}
\hline & RM & IC $95 \%$ & p \\
\hline \multicolumn{4}{|l|}{ Enfermedades del sistema circulatorio } \\
\hline Tabaquismo intenso & 1.83 & $1.1-2.8$ & $<0.01$ \\
\hline Tabaquismo intenso hombres & 2.5 & $1.4-4.4$ & $=0.001$ \\
\hline \multicolumn{4}{|l|}{ Tabaquismo 10 años antes de } \\
\hline morir hombres + de 70 años & 2.06 & $1.18-3.58$ & $=0.01$ \\
\hline \multicolumn{4}{|l|}{ Tabaquismo 1 año antes de morir } \\
\hline hombres + de 70 años & 1.9 & $1.05-3.70$ & $=0.03$ \\
\hline \multicolumn{4}{|l|}{ N eoplasias } \\
\hline Tabaquismo intenso & 2.88 & $1.1-7.02$ & $=0.01$ \\
\hline Tabaquismo intenso mujeres & 7.1 & $1.9-25.8$ & $=0.003$ \\
\hline \multicolumn{4}{|l|}{ Tabaquismo 1 año antes de morir } \\
\hline mujeres 35-69 años & 14.17 & $1.23-162.59$ & $9<0.01$ \\
\hline \multicolumn{4}{|l|}{ Tabaquismo 1 año antes de morir } \\
\hline mujeres + de 70 años & 7.24 & $1.71-30.53$ & $3=0.002$ \\
\hline \multicolumn{4}{|l|}{ Enfermedad pulmonar obstructiva crónica } \\
\hline Tabaquismo intenso & 4.17 & $1.9-9.1$ & $<0.001$ \\
\hline Tabaquismo intenso hombres & 2.9 & $1.8-8.1$ & $=0.03$ \\
\hline Tabaquismo intenso mujeres & 7.3 & $2.2-23.6$ & $=0.001$ \\
\hline $\begin{array}{l}\text { Tabaquismo } 1 \text { año antes de morir } \\
\text { mujeres + de } 70 \text { años }\end{array}$ & 4.82 & $1.41-16.50$ & $<0.01$ \\
\hline
\end{tabular}

También las mujeres de 70 años y más, fueron quienes tuvieron un riesgo aumentado hasta cinco veces de morir por enfermedad pulmonar obstructiva crónica (EPOC) cuando siguieron fumando hasta el año previo a su deceso $(R M=4.82 ; p<0.01$, IC $95 \%=1.41,16.50)$.

En el modelo de regresión para enfermedades del SR, se encontró que las personas que tuvieron positiva la variable tabaquismo intenso tuvieron una posibilidad 3.17 veces mayor de fallecer por enfermedades del SR, y el riesgo atribuible de esta variable para las enfermedades de este sistema en la población general fue de $76 \%$. $(R M=4.17 ; p<0.001$; IC $95 \%=1.9-9.1)$.

Al estratificar por edad y sexo, este mismo modelo mostró que en los hombres su posibilidad de fallecer por enfermedades del SR fue 1.9 veces mayor, y el riesgo atribuible de esta variable para las enfermedades del SR en la población general fue de $65 \%$. ( $R M=2.9$; $p=0.03$; IC 95\% = 1.1-8.1).

En el modelo de las mujeres, se encontró que las personas con variable positiva de tabaquismo intenso tuvieron una posibilidad 6.3 veces mayor de fallecer por enfermedades del SR, y el riesgo atribuible de esta variable para las enfermedades del SR en la población general fue de $86 \%$. $(R M=7.3 ; p=0.001$; IC $95 \%=2.2-23.6)$

\section{Discusión}

En este estudio se documentan las fracciones atribuibles para algunas causas de mortalidad con el consumo de tabaco, y tiene limitaciones que vale la pena considerar, como la posibilidad de un sesgo de memoria al preguntar sobre los hábitos de consumo de tabaco al familiar de alguna persona fallecida por enfermedades relacionadas con este consumo. Somos concientes de que los familiares de las personas que murieron por EPOC o CA de Pulmón es posible que recuerden mejor que sus familiares fumaban, a diferencia de los familiares de aquellos que murieron por otras enfermedades; sin embargo, la información obtenida en este estudio es consistente con lo emanado de otros estudios publicados en la literatura. ${ }^{8}$

A pesar de que las limitantes en el tamaño de la muestra en este estudio impiden hablar con seguridad de la frecuencia con la que se asociaron algunas variables con alguno de los padecimientos blanco en varios subgrupos estudiados, son de destacar las asociaciones encontradas entre fumar diario vs. cualquier otra frecuencia de consumo de tabaco y las enfermedades estudiadas en el análisis bivariado y en los modelos de regresión logística de los hombres. Aun cuando este estudio se llevó a cabo con una cantidad importante de los decesos de la delegación Coyoacán durante el año 
1998, algunas asociaciones hacen suponer que la muestra fue insuficiente para estimar el efecto del tabaquismo. Una de las limitaciones del estudio fue que no tuvimos poder estadístico para estudiar a las mujeres, y los resultados obtenidos también se ven afectados por el hecho de que la cohorte de fumadoras de México apenas está llegando a las edades de probabilidad de muerte.

La población encuestada presentó frecuencias de mortalidad para los padecimientos estudiados, similar a la publicada en el anuario de mortalidad de 1998, excepto en lo que se refiere a la mortalidad por neoplasias que en el anuario de 1998 representan para el Distrito Federal (DF) $13.3 \%$ de la proporción de fallecimientos, y nosotros sólo encontramos $2.8 \%$ de la población con neoplasias.

Este resultado pudo verse afectado por el hecho de que, como se ve en el cuadro I no tomamos todas las neoplasias, sólo aquellas que se asocian con el consumo de tabaco en la literatura consultada.

$\mathrm{Al}$ analizar los certificados de aquellas personas residentes en Coyoacán, que fallecieron de cualquiera de las causas que estudiamos en este trabajo, pero que no formaron parte de la encuesta, encontramos nuevamente que las frecuencias de presentación de los diagnósticos son similares a lo observado en el resto del D.F., excepto para EPOC, que en el anuario de 1998 del D.F. se encuentra representando $2.5 \%$ de la mortalidad y encontramos que en el SEED hay una proporción de $13.9 \%$ para las delegaciones políticas diferentes a Coyoacán. ${ }^{9}$

En el presente estudio se encontró que $2.8 \%$ de las muertes estudiadas se debió a alguna neoplasia, y de éstos $45.5 \%$ fueron fumadores.

En la Encuesta Nacional de Adicciones (ENA) de 1998 se encontró una prevalencia de fumadores de $27.7 \%$ en el ámbito nacional de entre 12 y 65 años de edad; nosotros encontramos una prevalencia general de consumo de tabaco en por lo menos los últimos 10 años de $29 \%$; la prevalencia de tabaquismo en hombres según la ENA fue del $42.9 \%$; sin embargo, encontramos $46.3 \%$ en la población estudiada, y la de mujeres de acuerdo a la ENA fue de $16.3 \%$; nosotros encontramos $16.3 \%$; por lo tanto consideramos que no existe un sesgo de selección que invalide nuestro estudio. ${ }^{10-13}$

Este primer estudio muestra la utilidad de los sistemas de vigilancia de la Secretaría de Salud, tales como el SEED, para llevar a cabo trabajos de investigación y además llama la atención sobre la necesidad de más trabajos orientados a estudiar las variables que se con- sideraron, replanteándolas nuevamente como una propuesta para otros que reconsideren los procedimientos utilizados para estudiar mortalidad por enfermedades crónicas y los factores de riesgo relacionados con ellas en poblaciones latinas.

Nuestros resultados resaltan la importancia de realizar estudios adicionales para definir con mayor precisión las fracciones de mortalidad atribuibles al tabaquismo; estudios futuros requerirán de un mayor tamaño de la muestra para poder identificar, con mayor precisión, las fracciones de mortalidad por sexo y grupo de edad. Sin embargo, nuestros resultados concuerdan con lo publicado en otras series y sugieren que el tabaquismo en México es causa de una fracción importante de enfermedades.

\section{Referencias}

1. Secretaría de Salud. Consejo N acional contra las A dicciones. Programa contra el tabaquismo. México, D.F.: SSA, 1992.11-32.

2. Kuri-Morales, Pablo. Tabaquismo: una epidemia mundial. [Boletín] Epidemiología Sistema N acional de Vigilancia Epidemiológica. 1995;12: 12, semana 35.

3. Secretaría de Salud. Consejo N acional contra las Adicciones. Situación actual de las adicciones. México: SSA, 1995. 3-43.

4. Tapia-C onyer T, Kuri-Morales P, Meneses-González F. SmokingAttributable Mortality-México, 1992. MMW R. 1995 Mayo; 44:372-378.

5. Samet JM. Los riesgos del tabaquismo activo y pasico. Salud Publica Mex 2002;44 supl 1:S144-S160

6. Chronic Disease and Health Promotion Adapted from MMW R, Tobacco Topics. Cigarrette Smoking-A tributable Mortality and Years of Potencial Life Lost. EUA. 1990. pp 77-81.

7. O rganización Panamericana de la Salud-0 rganización Mundial de la Salud. Clasificación Estadística Internacional de Enfermedades y Problemas relacionados con la salud. D écima revisión CIE-10. Ginebra O PS-O MS, 1995. 8. Whittemore AS. Estimating attributable risk from case-control studies. Am. J. Epidemiol. 1982;117:76-85

9. Peto R, LópezAD, Boreham J,Thun M, Heath C. Mortality from tobacco in developed countries: indirect estimation from national vital statistics. Lancet 1992;339:1268-78.

10. Secretaría de Salud. Dirección General de Estadística e informática. Mortalidad 1998. México: SSA, 2000: "Principales causas de mortalidad general Distrito Federal", 101.

11. Secretaría de Salud. Dirección General de Epidemiología. Encuesta $\mathrm{N}$ acional de Adicciones Tomo II Tabaco. México: SSA, 1994. 21-86.

12. Secretaría de Salud. Consejo Nacional Contra las Adicciones. Información básica sobre tabaquismo. México: SSA, 1995. 3-30 51-54.

13. Secretaría de Salud. Dirección General de Epidemiología. El Consumo de Tabaco en México y Encuesta $\mathrm{N}$ acional de Adicciones 1998. México: SSA, 2000. 187-195. 